\title{
Diversity of plant knowledge in a "Caiçara" community from the Brazilian Atlantic Forest coast ${ }^{1}$
}

\author{
Mariana Reis de Brito ${ }^{2,3}$ and Luci de Senna-Valle ${ }^{2}$
}

Recebido em 18/05/2012. Aceito em 13/06/2012

\begin{abstract}
RESUMO
(Diversidade do conhecimento de plantas em uma comunidade "Caiçara" da Mata Atlântica brasileira). Há um número crescente de publicações sobre populações Caiçaras, o que revela o interesse dos pesquisadores sobre esses grupos. Este estudo teve como objetivo investigar a flora utilizada pelos especialistas da comunidade Caiçara da Praia do Sono, registrando a forma de uso desses táxons e procurando perceber os sistemas tradicionais de manejo que visam conservar os ecossistemas naturais. Doze informantes foram selecionados e entrevistados. A análise de agrupamento realizada juntamente com o teste qui-quadrado evidenciaram que o conhecimento etnobotânico mostra-se heterogeneamente distribuído, ao se considerar o gênero dos entrevistados. 190 táxons foram indicados e encontram-se ordenados em 9 categorias de usos. O índice de Shannon-Wiener $\left(H^{\prime}\right)$ obtido neste estudo foi o segundo mais alto quando comparado com o das demais comunidades costeiras do Brasil. Este trabalho constatou que os informantes da Praia do Sono preservam um amplo conhecimento sobre os recursos vegetais que os cercam e com os quais mantêm grande intimidade. Este conhecimento não é só importante, mas fundamental nas discussões sobre a aplicação de usos sustentáveis e estratégias de conservação para esta área.
\end{abstract}

Palavras-chave: Mata Atlântica, Caiçaras, Conservação, Etnobotânica, Plantas úteis

\begin{abstract}
(Diversity of plant knowledge in a "Caiçara" community from the Brazilian Atlantic Forest coast). The number of publications about the Caiçaras population is growing, which shows that researchers are interested in these natives. This study aimed to survey the flora used by local specialists of the Praia do Sono Caiçara community, and recorded how these taxa were used, with the goal of understanding traditional management systems that help to conserve natural ecosystems. Twelve informants were selected and interviewed. The applied grouping analysis, together with the chi-squared test, underlined that the analysed ethnobotanical knowledge showed a heterogeneous distribution in relation to the gender of the interviewee. A total of 190 taxa were cited and were classified into nine usage categories. The Shannon-Wiener index $\left(H^{\prime}\right)$ value obtained in this study was the second highest in comparison to other Brazilian coastal communities. This work showed that the local specialists of this Caiçara community maintain a wide knowledge of, and affinity to, the plant resources that surround them. This knowledge is not only important, but fundamental to discussions about the application of sustainable use and management strategies for this area of conservation value.
\end{abstract}

Keywords: Atlantic Forest, Caiçaras, Conservation, Ethnobotany, Useful plants

\section{Introduction}

Since the discovery of Brazil, the Atlantic Forest has been influenced by a large number of human disturbances (Tabarelli et al. 2010). The dramatic devastation of the richness and diversity of this region has, over the years, promoted the disappearance of several species and caused hundreds of others to come under pressure of extinction (Ribeiro et al. 2009). Because of its indisputable importance for human survival, combined with the numerous threats that it has been suffering from, the conservation of the biodiversity of the Atlantic Forest has been the focus of several studies, whose arguments in its defence include environmental, political-economic, ethnic, aesthetic and utilitarian dimensions (Hanazaki 2003). According to Diegues \& Arruda (2001), the knowledge of local populations is, in some countries,

\footnotetext{
${ }^{1}$ This study is part of the Master's dissertation of the first Author

${ }^{2}$ Universidade Federal do Rio de Janeiro, Museu Nacional, Departamento de Botânica, Rio de Janeiro, RJ, Brazil

${ }^{3}$ Author for correspondence: marianareis2002@hotmail.com
} 
considered to be the primary focus in discussions about conservation issues.

This research was conducted in a community that is recognized as belonging to the "Caiçara cultural type". The Caiçara culture appeared in the Atlantic Forest, more specifically the coastal states of São Paulo, Rio de Janeiro and Paraná, as a result of miscegenation between indigenous populations, Portuguese colonizers and, to a lesser degree, Africans slaves (Sanches 2004). Survival of the family unit is based on the activities of cutting and burning, itinerant agriculture, fishing and extraction of resources from the environment (Begossi 1998). According to Hanazaki (2003), these natives play a major role in the conservation of the Atlantic Forest, due to their vast knowledge of the environment, which has accumulated over many generations and could be transformed into a valuable ally in the search for improving the quality of life of several populations.

This study aimed to survey the flora used by local specialists of the Praia do Sono Caiçara community, by recording how these taxa are used, with the goal of understanding traditional management systems that help to conserve natural ecosystems. Taking into account that the study group interacts directly with the Atlantic Forest biome, accumulating extensive knowledge about the native plant species, we sought to analyse and to describe how knowledge about the use of plants is distributed among the interviewees, and to group the cited species into categories of usage.

\section{Materials and methods}

\section{Study area}

The Praia do Sono Caiçara community is located on a beach called Praia do Sono, inside the Ecological Reserve of Juatinga, which is within the Environmental Preservation Area of Cairuçu, Paraty Municipality, in the state of Rio de Janeiro. The community is located at the coordinates $23^{\circ} 19^{\prime}$ $55.2^{\circ} \mathrm{S}$ and $44^{\circ} 37^{\prime} 57.8^{\circ} \mathrm{W}$ and occupies an area that is 1365 metres long.

The Ecological Reserve of Juatinga is home to twelve traditional populations, distributed along the coast in five main centres: Praia do Sono, Ponta Negra, Praia Grande do Pouso da Cajaíba, Ponta da Juatinga, and Mamanguá (SEMADS-RJ 2001).

\section{Data collection}

The fieldwork was undertaken between February 2008 and June 2009, with monthly inspections. The "snowball" technique was applied in order to get a list of local specialist informants. The data were obtained through the techniques of "direct observation", "participatory observation" and "semi-structured interviews" (Albuquerque et al. 2008).

Samplings were carried out in the woods, guided by five of the six local men who participated in the survey, using the forest sections most frequented by them (Alexiades \&
Sheldon 1996). The useful species present on the beach, and those cultivated in the backyards of the local informants were also collected; however, these areas were covered for all 12 informants interviewed.

All botanical material collected was deposited in the herbarium of Museu Nacional do Rio de Janeiro (R). The identification of botanical material was undertaken using the literature, by comparison with specimens from the herbaria at the Museu Nacional (R) and Jardim Botânico do Rio de Janeiro (RB), as well as with the help of specialists.

\section{Data analysis}

Level of similarity of knowledge between genders was assessed using cluster analysis based on the known species provided by each informant. Sorensen's coefficient was calculated, and the connection method UPGMA was used. Informants' knowledge of plant diversity by gender was statistically compared using the $t$-test.

The chi-square test was used to evaluate if the difference in knowledge about the uses of plants between genders was significant.

In order to classify the origin of the plants collected, we adopted the following definitions: native plants (those belonging to the Atlantic Forest domain) and exotic plants (species originating in other areas). For this purpose we consulted Corrêa (1926-1978) and Stehmann et al. (2009).

Sampling effort was measured by adjustment of the straight simple linear regression line calculated from the accumulation of species' richness (Whittaker 1975), according to the cumulative number of observations (Loss \& Silva 2005) corresponding to the sequential insertion of interviews.

The Shannon-Wiener diversity index (Begossi 1996) was calculated to compare data from this study with results originating from ethnobotanical research carried out in different communities along the Brazilian coast.

The uses mentioned were categorized according to those proposed by Prance et al. (1987) and Phillips \& Gentry (1993), with a few adaptations to fit the context of the present study, but always respecting the information provided by the local specialist interviewed and attempting to minimize potential distortions. The uses mentioned by the informants were classified into nine general categories (Table 1).

All statistical analyses were performed using the program PAST v1.34 (Hammer et al. 2001).

\section{Results and discussion}

\section{Knowledge distribution}

Twelve informants $(n=12)$ were interviewed, each with knowledge of the local vegetation. The informants were between 29 and 88 years old and there were six men and six women. Ten are native to Praia do Sono, and two came from surrounding regions but have resided in the community for over 50 years. 
Table 1. The usage categories of the species indicated by local specialists of the Praia do Sono Caiçara community and their corresponding descriptions.

\begin{tabular}{|c|c|}
\hline Usage Categories & Description Categories \\
\hline Food & Cited as "plant to eat" and "plant for seasoning" \\
\hline Construction & Used on house building, ranch building (rafters, sleepers, roof shingles, mooring, etc.) and fence posts \\
\hline Fuelwood & Cited as "wood to burn" \\
\hline Medicinal & Cited as "plant to remedy" \\
\hline Ornamental & Plants used to decorate backyards and homes \\
\hline Ritualistic & Plants used in baths to "take of the evil eye" and "open the way" and cultivated around homes to "drive away the evil eye" \\
\hline Tecnological & Used in the creation of objects for domestic use, recreational or to build furniture, canoes, etc \\
\hline Veterinary & Used for infestation of lice in farmed animals \\
\hline Others & Its known good for "shade", "bird food" and "repellent" \\
\hline
\end{tabular}

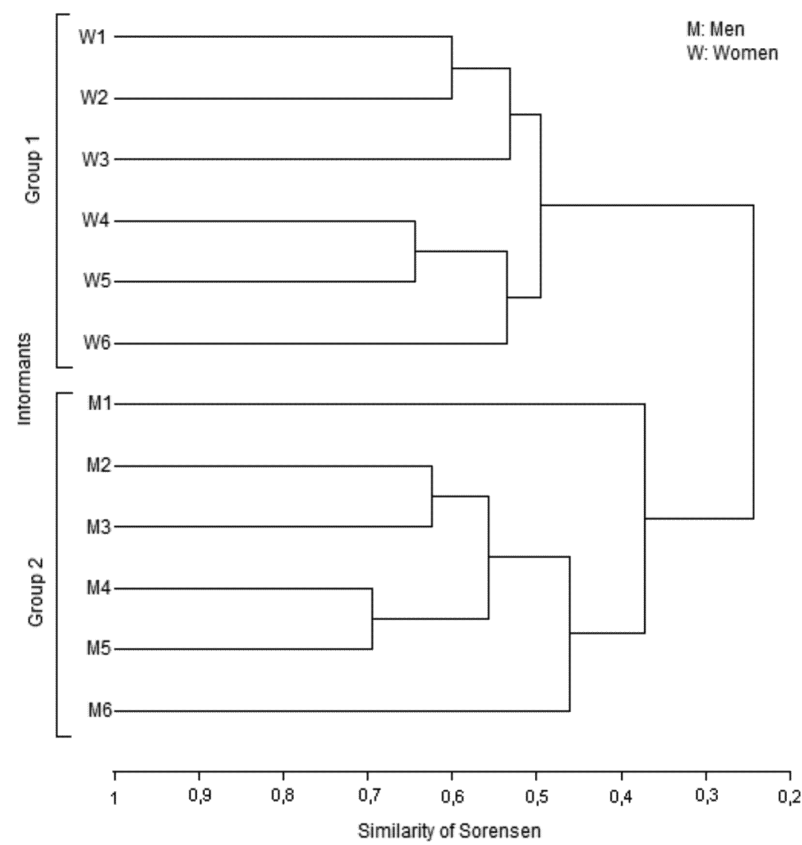

Figure 1. Analysis of similarity of knowledge regarding the use of plant resources among the informants of the Praia do Sono Caiçara community.

Investigation of similarities in terms of knowledge about plant resource uses, from the dendrogram produced from the matrix of presence/absence of each species mentioned by each informant, showed a significant adjustment to the calculated matrix of coefficients ( $\mathrm{rcs}=0.8944$ ) and formed two separate groups clearly separating the genders. This indicated that there is a preference in the use of certain species by both men and women (Fig. 1). However, when the diversity of these plants was evaluated between both genders, using the $t$-test, this difference did not appear significant $(P=0.2507)$. Therefore, men and women have some preference regarding plant use, and share similar values about plant diversity.

The first group shown in the dendrogram consisted of the six women interviewed; the main activities carried out by them are related to maintenance and home care, including child rearing, food preparation, cleaning and tidying the house, attention to the backyard and managing small livestock. The role they play in the social organization of the community gives them a better understanding of the species used for medicinal purposes, food, ornamental and veterinary purposes, these being the factors responsible for this grouping. The second group was formed of the six men who participated in the survey, who are responsible for functions related to hunting and fishing, construction of houses, supply of firewood, etc. These specialists stand out in terms of knowledge about plants native to the Atlantic Forest, especially those with an arboreal habit included in the usage categories construction, fuelwood and technological. The results corroborate with Laraia (2002), affirming that humans are shaped by the cultural environment in which they are socialized. As recorded by Miranda \& Hanazaki (2008), the cluster analysis yielded promising results for the understanding of indigenous knowledge about plants, explaining the intricacies and subtleties that are present in the relations between the studied group and the plant resources.

A chi-square test verified that there is a difference between genders in knowledge about the use of plants $\left(X^{2}=477.47\right.$; $\mathrm{df}=8 ; P<0.0001)$. The main reason for this difference is the large number of species utilized in the usage categories for medicinal and veterinary purposes cited by female informants, and in the significant number of species in the construction, fuelwood and technological categories, cited by male informants (Fig. 2). Hanazaki et al. (2000) studied two Caiçaras communities in São Paulo state, and also showed that knowledge about plants differs according to the gender, especially in relation to plants used in the technological category.

\section{Data on plant species}

We obtained indications of 215 ethnospecies for various purposes. From this, a total of 190 taxa were identified (Tab. 2). The plants noted in Table 2 are distributed across 61 botanical families, with the highest number of species being represented by Fabaceae (14 spp.), Poaceae (13 spp.), Lamiaceae (11 spp.) and Myrtaceae (11 spp.).

Considering the origin of the species, the informants in the present study mentioned 108 native species and 82 exotic species. Miranda \& Hanazaki (2008) studied the 
Table 2. List of species cited as useful by the local specialists of the Praia do Sono Caiçara community. Family/Scientific name. Local name. Habit: Ar-arboreal, Hb-herb, Sh-shrub, Vi-vine. Usage categories: food-food, cons-construction, fue-fuelwood, med-medicinal, orn-ornamental, rit-ritualistic, tec- tecnological, vet-veterinary, oth-others/ Part used: ap-aerial part, c-crown, fl-flower, fr-fruit, i-inflorescence, l-leaf, m-mucilage, r-root, s-seed, st-stem, wp-whole plant. Origin and Collection location (O/Loc): N-native to the Atlantic Forest domain, E-exotic to the biome, B-backyards, F-forest, Bea-beach.

\begin{tabular}{|c|c|c|c|c|}
\hline Family/Scientific name & Local name & Habit & Usage categories / Part used & $\mathrm{O} / \mathrm{Loc}$ \\
\hline \multicolumn{5}{|l|}{ Alismataceae } \\
\hline Echinodorus grandiflorus Mitch. & chapéu-de-couro & $\mathrm{Hb}$ & med (l) & $\mathrm{N} / \mathrm{B}$ \\
\hline \multicolumn{5}{|l|}{ Amaranthaceae } \\
\hline Alternanthera brasiliana (L.) O. Kuntze & terramicina & $\mathrm{Hb}$ & med (l) & $\mathrm{N} / \mathrm{B}$ \\
\hline Iresine herbstii Hook. & arnica; paraguaia & $\mathrm{Hb}$ & med; orn (l) & $\mathrm{E} / \mathrm{B}$ \\
\hline \multicolumn{5}{|l|}{ Amaryllidaceae } \\
\hline Allium cepa L. & cebola & $\mathrm{Hb}$ & food (st) & $\mathrm{E} / \mathrm{B}$ \\
\hline Allium fistulosum L. & cebolinha & $\mathrm{Hb}$ & food (l) & $\mathrm{E} / \mathrm{B}$ \\
\hline Allium sativum $L$. & alho & $\mathrm{Hb}$ & food; med (st) & $\mathrm{E} / \mathrm{B}$ \\
\hline \multicolumn{5}{|l|}{ Anacardiaceae } \\
\hline Anacardium occidentale L. & cajú & Sh & food; med (fr) & $\mathrm{N} / \mathrm{B}$ \\
\hline Mangifera indica $\mathrm{L}$. & manga & Sh & food (fr); med (l) & $\mathrm{E} / \mathrm{B}$ \\
\hline Schinus terebinthifolius Raddi & aroeira & Sh & cons; med; tec (st); oth (fr) & N/Bea \\
\hline Spondias mombin $\mathrm{L}$ & cajá & Sh & food $(\mathrm{fr})$ & $\mathrm{N} / \mathrm{B}$ \\
\hline Tapirira guianensis Aubl. & canafístula; cupiuva & Sh & cons; tec (st) & $\mathrm{N} / \mathrm{F}$ \\
\hline \multicolumn{5}{|l|}{ Annonaceae } \\
\hline Guatteria australis A.St.-Hil. & bicuíba & Sh & med (fr) & $\mathrm{N} / \mathrm{F}$ \\
\hline Rollinia mucosa (Jacq.) Baill. & condessa & Sh & food (fr) & $\mathrm{N} / \mathrm{B}$ \\
\hline \multicolumn{5}{|l|}{ Apiaceae } \\
\hline Coriandrum sativum L. & coentro & $\mathrm{Hb}$ & food (1) & $\mathrm{E} / \mathrm{B}$ \\
\hline Eryngium foetidum $\mathrm{L}$. & coentro-bravo; coentrão & $\mathrm{Hb}$ & food (l) & $\mathrm{N} / \mathrm{B}$ \\
\hline Petroselinum crispum (Mill.) A. W. Hill & salsa & $\mathrm{Hb}$ & food (l) & $\mathrm{E} / \mathrm{B}$ \\
\hline \multicolumn{5}{|l|}{ Apocynaceae } \\
\hline Catharanthus roseus (L.) G. Don & boa-noite & $\mathrm{Hb}$ & orn (ap) & $\mathrm{E} / \mathrm{B}$ \\
\hline \multicolumn{5}{|l|}{ Araceae } \\
\hline Anthurium sp. & cipó-imbé & $\mathrm{Vi}$ & cons; tec (st) & $\mathrm{N} / \mathrm{F}$ \\
\hline Colocasia esculenta Schott & inhame & $\mathrm{Hb}$ & food (st) & $\mathrm{E} / \mathrm{B}$ \\
\hline Heteropsis salicifolia Kunth & timbupeba & $\mathrm{Vi}$ & cons; tec (st) & $\mathrm{N} / \mathrm{F}$ \\
\hline \multicolumn{5}{|l|}{ Arecaceae } \\
\hline Astrocaryum aculeatissimum (Schott) Burret & côco-preto & Sh & food (fr) & $\mathrm{N} / \mathrm{F}$ \\
\hline Attalea dubia (Mart.) Burret & indaiá & Sh & food (fr) & $\mathrm{N} / \mathrm{F}$ \\
\hline Bactris setosa Mart. & côco-de-natal; tucum & Sh & food (fr) & $\mathrm{N} / \mathrm{F}$ \\
\hline Cocos nucifera $\mathrm{L}$. & côco & Sh & food (fr) & $\mathrm{E} / \mathrm{B}$ \\
\hline Euterpe edulis Mart. & jiçara; juçara & Sh & food; cons (st) & $\mathrm{N} / \mathrm{F}$ \\
\hline Syagrus pseudococus (Raddi) Glassman & pati & Sh & cons; tec (st) & $\mathrm{N} / \mathrm{F}$ \\
\hline \multicolumn{5}{|l|}{ Asparagaceae } \\
\hline Aloe vera (L.) Burm. f. & babosa & $\mathrm{Hb}$ & $\operatorname{med}(\mathrm{m})$ & $\mathrm{E} / \mathrm{B}$ \\
\hline \multicolumn{5}{|l|}{ Asteraceae } \\
\hline Achyrocline satureoides (Lam.) DC. & camomila; macela & $\mathrm{Hb}$ & med (i) & $\mathrm{N} / \mathrm{F}$ \\
\hline Ageratum conyzoides $\mathrm{L}$. & erva-de-são-joão & $\mathrm{Hb}$ & med (wp) & $\mathrm{N} / \mathrm{B}$ \\
\hline Baccharis dracunculifolia DC. & vassoura-de-alecrim & $\mathrm{Hb}$ & tec (ap) & $\mathrm{N} / \mathrm{F}$ \\
\hline Baccharis trimera (Less.) DC. & carqueja & $\mathrm{Hb}$ & med (st) & $\mathrm{E} / \mathrm{B}$ \\
\hline Bidens pilosa $\mathrm{L}$. & picão & $\mathrm{Hb}$ & med (wp) & $\mathrm{N} / \mathrm{B}$ \\
\hline Lactuca sativa $\mathrm{L}$. & alface & $\mathrm{Hb}$ & food (l) & $\mathrm{E} / \mathrm{B}$ \\
\hline Mikania glomerata Spreng. & guaco & $\mathrm{Vi}$ & $\operatorname{med}(1)$ & $\mathrm{N} / \mathrm{B}$ \\
\hline Vernonia beyrichii Less. & cambará-roxo & Sh & med (l) & $\mathrm{N} / \mathrm{F}$ \\
\hline Vernonia polyanthes Less. & assa-peixe & Ar & med (l) & $\mathrm{E} / \mathrm{B}$ \\
\hline \multicolumn{5}{|l|}{ Bignoniaceae } \\
\hline Crescentia cujete L. & cabaço & Ar & fue (st); med; tec (fr) & $\mathrm{E} / \mathrm{B}$ \\
\hline Tabebuia cassinoides (Lam.) DC. & caxeta & Ar & tec $(s t)$ & $\mathrm{N} / \mathrm{F}$ \\
\hline Tabebuia heptaphylla (Vell.) Toledo & ipê & Ar & cons; tec (st) & $\mathrm{N} / \mathrm{F}$ \\
\hline Sparattosperma leucanthum (Vell.) K. Schum & ipê-da-capoeira & Ar & cons (st) & $\mathrm{N} / \mathrm{F}$ \\
\hline
\end{tabular}


Table 2. Continuation.

\begin{tabular}{|c|c|c|c|c|}
\hline Family/Scientific name & Local name & Habit & Usage categories / Part used & $\mathrm{O} / \mathrm{Loc}$ \\
\hline \multicolumn{5}{|l|}{ Boraginaceae } \\
\hline Cordia verbenacea DC. & baleeira & Sh & med (l) & $\mathrm{E} / \mathrm{B}$ \\
\hline \multicolumn{5}{|l|}{ Brassicaceae } \\
\hline Brassica oleracea L. var. acephala D.C. & couve & $\mathrm{Hb}$ & food; med (l) & $\mathrm{E} / \mathrm{B}$ \\
\hline \multicolumn{5}{|l|}{ Bromeliaceae } \\
\hline Ananas comosus (L.) Merr. & abacaxi & $\mathrm{Hb}$ & food; med (fr) & $\mathrm{E} / \mathrm{B}$ \\
\hline \multicolumn{5}{|l|}{ Caricaceae } \\
\hline Carica papaya L. & mamão-macho & $\mathrm{Ar}$ & $\operatorname{med}(\mathrm{fl})$ & $\mathrm{E} / \mathrm{B}$ \\
\hline \multicolumn{5}{|l|}{ Cecropiaceae } \\
\hline Cecropia pachystachya Trec. & baibera; imbaíba & $\mathrm{Ar}$ & fue; tec (st); med (l) & $\mathrm{N} / \mathrm{F} ; \mathrm{B}$ \\
\hline \multicolumn{5}{|l|}{ Chenopodiaceae } \\
\hline Chenopodium ambrosioides $\mathrm{L}$. & santa-maria & $\mathrm{Hb}$ & med; vet (ap) & $\mathrm{E} / \mathrm{B}$ \\
\hline \multicolumn{5}{|l|}{ Chrysobalanaceae } \\
\hline Hirtella hebeclada Moric. Ex DC. & simbiíba; simbiúva & $\mathrm{Ar}$ & cons; fue (st) & $N / F$ \\
\hline \multicolumn{5}{|l|}{ Clusiaceae } \\
\hline Clusia lanceolata Cambess. & cebola-da-praia & $\mathrm{Ar}$ & orn (ap); oth (fr) & $\mathrm{N} / \mathrm{F}$ \\
\hline \multicolumn{5}{|l|}{ Combretaceae } \\
\hline Terminalia catappa $\mathrm{L}$. & amendoeira & $\mathrm{Ar}$ & food (fr); fue; cons (st); oth (c) & E/Bea \\
\hline \multicolumn{5}{|l|}{ Convovulaceae } \\
\hline Ipomea batatas (L.) Lam. & batata-doce & $\mathrm{Hb}$ & food; med (r) & $\mathrm{E} / \mathrm{B}$ \\
\hline \multicolumn{5}{|l|}{ Costaceae } \\
\hline Costus spicatus (Jacq.) Sw. & cana-do-brejo & $\mathrm{Hb}$ & med (l) & N/B \\
\hline \multicolumn{5}{|l|}{ Crassualceae } \\
\hline Bryophyllum pinnatum (L. f.) Oken & estalo & $\mathrm{Hb}$ & med (l) & $\mathrm{E} / \mathrm{F} ; \mathrm{B}$ \\
\hline Kalanchoe brasiliensis Cambess & saião & $\mathrm{Hb}$ & med (l) & N/B \\
\hline \multicolumn{5}{|l|}{ Crucifera } \\
\hline Nasturtium officinale R. Br. & agrião & $\mathrm{Hb}$ & food; med (l) & $\mathrm{E} / \mathrm{B}$ \\
\hline \multicolumn{5}{|l|}{ Cucurbitaceae } \\
\hline Cucumis sativus $\mathrm{L}$. & pepino & $\mathrm{Hb}$ & food (fr) & $\mathrm{E} / \mathrm{B}$ \\
\hline Cucurbita pepo L. & abóbora & $\mathrm{Hb}$ & food (fr); med (fl) & $\mathrm{E} / \mathrm{B}$ \\
\hline Momordica charantia $\mathrm{L}$. & melão-de-são-caetano & $\mathrm{Vi}$ & med (l) & $\mathrm{E} / \mathrm{B}$ \\
\hline Sechium edule (Jacq.) Sw. & chuchu & $\mathrm{Vi}$ & food (fr); med (l) & $\mathrm{E} / \mathrm{B}$ \\
\hline \multicolumn{5}{|l|}{ Dilleniaceae } \\
\hline Davilla rugosa Poiret & cipó-caboclo & $\mathrm{Vi}$ & cons; med; tec (st) & $\mathrm{N} / \mathrm{F}$ \\
\hline \multicolumn{5}{|l|}{ Elaeocarpaceae } \\
\hline Sloanea guianensis Benth. & guariçica-da-folha-grande & $\mathrm{Ar}$ & cons (st) & $\mathrm{N} / \mathrm{F}$ \\
\hline \multicolumn{5}{|l|}{ Erytroxylaceae } \\
\hline Erytroxylum ovalifolium peyr. & futiabeira & $\mathrm{Ar}$ & med (l) & N/B \\
\hline \multicolumn{5}{|l|}{ Euphorbiaceae } \\
\hline Alchornea glandulosa Poepp. \& Endl. & chichá & $\mathrm{Ar}$ & tec (st) & $\mathrm{N} / \mathrm{F}$ \\
\hline Aleurites moluccana Willd. & noga & $\mathrm{Ar}$ & food; tec (fr) & $\mathrm{E} / \mathrm{B}$ \\
\hline Euphorbia prostrata Aiton & quebra-pedra & $\mathrm{Hb}$ & med (wp) & N/B \\
\hline Euphorbia pulcherrima Willd. ex Klotzsch & seis-meis & Sh & orn (ap) & $\mathrm{E} / \mathrm{B}$ \\
\hline Hyeronima alchorneoides Fr. Allem. & arecurana; aricurana & $\mathrm{Ar}$ & cons; tec (st) & $\mathrm{N} / \mathrm{F}$ \\
\hline Mabea brasiliensis Müll. Arg. & canudo & $\mathrm{Ar}$ & cons (st) & $\mathrm{N} / \mathrm{F}$ \\
\hline Manihot esculenta Crantz & ipi; mandioca & $\mathrm{Hb}$ & food (r) & $\mathrm{N} / \mathrm{B}$ \\
\hline Pera glabrata (Schott) Baill. & chile; casca-preta & $\mathrm{Ar}$ & fue; cons (st) & $\mathrm{N} / \mathrm{F}$ \\
\hline Ricinus communis $\mathrm{L}$. & mamona & Sh & food; tec (fr); med (l) & $\mathrm{E} / \mathrm{B}$ \\
\hline Sapium sp. & tarumã & Ar & tec (st) & $\mathrm{N} / \mathrm{F}$ \\
\hline
\end{tabular}


Table 2. Continuation

\begin{tabular}{|c|c|c|c|c|}
\hline Family/Scientific name & Local name & Habit & Usage categories / Part used & $\mathrm{O} / \mathrm{Loc}$ \\
\hline \multicolumn{5}{|l|}{ Fabaceae } \\
\hline Cajanus cajan (L.) Millsp. & feijão-guandu & Sh & food (fr); med (l) & $\mathrm{E} / \mathrm{B}$ \\
\hline Desmodium adscendens (SW.) DC. & carrapichinho & $\mathrm{Hb}$ & med (wp) & $\mathrm{N} / \mathrm{F}$ \\
\hline Hymenaea courbaril $\mathrm{L}$. & jatobá & $\mathrm{Ar}$ & cons; med (st) & $\mathrm{N} / \mathrm{F}$ \\
\hline Inga lanceifolia Benth. & cabriúba; cambriúba & $\mathrm{Ar}$ & cons $(\mathrm{st})$ & $\mathrm{N} / \mathrm{F}$ \\
\hline Inga striata Benth. & ingá & $\mathrm{Ar}$ & food (fr); cons (st) & $\mathrm{N} / \mathrm{F}$ \\
\hline Inga vera Willd. & ingá & $\mathrm{Ar}$ & food (fr); cons (st) & $\mathrm{N} / \mathrm{F}$ \\
\hline Mimosa pudica L. & dormideira & $\mathrm{Hb}$ & med; rit (wp) & $\mathrm{N} / \mathrm{B}$ \\
\hline Myrocarpus frondosus Fr. Allem. & cabriúba-vermelha & $\mathrm{Ar}$ & cons; tec (st) & $\mathrm{N} / \mathrm{F}$ \\
\hline Phaseolus vulgaris $\mathrm{L}$. & feijão & $\mathrm{Hb}$ & food (fr) & $\mathrm{E} / \mathrm{B}$ \\
\hline Piptadenia gonoacantha (Mart.) Macbr. & cobi & $\mathrm{Ar}$ & tec (st) & $\mathrm{N} / \mathrm{F}$ \\
\hline Schizolobium parahyba (Vell.) Blake & garapubu & $\mathrm{Ar}$ & tec (st) & $\mathrm{N} / \mathrm{F}$ \\
\hline Senna multijuga Rich. I. \& B. & aleluia & $\mathrm{Ar}$ & fue (st); orn (wp) & $\mathrm{N} / \mathrm{F}$ \\
\hline Swartzia myrtifolia var. elegans (Schott) R. S. Cowan & laranjeira-do-mato & $\mathrm{Ar}$ & cons $(s t)$ & $\mathrm{N} / \mathrm{F}$ \\
\hline Tachigali paratyensis (Vell.) H. C. Lima & ingá-de-flecha & $\mathrm{Ar}$ & tec (st) & $\mathrm{N} / \mathrm{F}$ \\
\hline \multicolumn{5}{|l|}{ Lamiaceae } \\
\hline Leonotis nepetaefolia (L.) R. Br. & cordão-de-frade & $\mathrm{Hb}$ & med (l); rit (wp) & $\mathrm{E} / \mathrm{B}$ \\
\hline Leonurus sibiricus $\mathrm{L}$. & santa-rita & $\mathrm{Hb}$ & med (wp) & $\mathrm{E} / \mathrm{B}$ \\
\hline Marsypianthes chamaedrys (Vahl.) Kuntze & erva-madre & $\mathrm{Hb}$ & med (wp) & $\mathrm{N} / \mathrm{B}$ \\
\hline Mentha pulegium $\mathrm{L}$. & poejo & $\mathrm{Hb}$ & med (wp) & $\mathrm{E} / \mathrm{B}$ \\
\hline Mentha sativa $\mathrm{L}$. & $\begin{array}{c}\text { hortelã-de-bicho; } \\
\text { hortelã-miúdo; hortelã }\end{array}$ & $\mathrm{Hb}$ & food; med (l) & $\mathrm{E} / \mathrm{B}$ \\
\hline Ocimum basilicum $\mathrm{L}$. & manjericão & $\mathrm{Hb}$ & food; med (l) & $\mathrm{E} / \mathrm{B}$ \\
\hline Ocimum selloi Benth. & alfavaca & $\mathrm{Hb}$ & food; med (l) & $\mathrm{N} / \mathrm{B}$ \\
\hline Plectranthus amboinicus (Lour.) Spreng. & hortelã-de-galinha & $\mathrm{Hb}$ & food; med (l) & $\mathrm{E} / \mathrm{B}$ \\
\hline Plectranthus barbatus Andrews & boldo & $\mathrm{Hb}$ & med (l) & $\mathrm{E} / \mathrm{B}$ \\
\hline Plectranthus ornatus Codd. & anador & $\mathrm{Hb}$ & med (l) & $\mathrm{E} / \mathrm{B}$ \\
\hline Rosmarinus officinalis $\mathrm{L}$. & alecrim & $\mathrm{Hb}$ & food; med (l) & $\mathrm{E} / \mathrm{B}$ \\
\hline \multicolumn{5}{|l|}{ Lauraceae } \\
\hline Laurus nobilis L. & louro & $\mathrm{Ar}$ & food; med (l) & $\mathrm{E} / \mathrm{B}$ \\
\hline Ocotea sp. & canela & $\mathrm{Ar}$ & cons $(\mathrm{st})$ & $\mathrm{N} / \mathrm{F}$ \\
\hline Persea americana Mill. & abacate & $\mathrm{Ar}$ & food (fr); med (l) & $\mathrm{E} / \mathrm{B}$ \\
\hline \multicolumn{5}{|l|}{ Lecythidaceae } \\
\hline Cariniana estrellensis (Raddi) Kuntze & jequitibá & $\mathrm{Ar}$ & med; tec (st) & $\mathrm{N} / \mathrm{F}$ \\
\hline Couratari pyramidata (Vell.) R. Knuth & sapucaia-de-esqueiro & $\mathrm{Ar}$ & cons (st); tec (r) & $\mathrm{N} / \mathrm{F}$ \\
\hline Lecythis pisonis Camb. & sapucaia-de-côco & $\mathrm{Ar}$ & cons $(\mathrm{st})$ & $\mathrm{N} / \mathrm{F}$ \\
\hline \multicolumn{5}{|l|}{ Loranthaceae } \\
\hline Struthanthus concinnus Mart. & erva-de-passarinho & $\mathrm{Vi}$ & med (l) & $\mathrm{N} / \mathrm{B}$ \\
\hline \multicolumn{5}{|l|}{ Lytraceae } \\
\hline Punica granatum $\mathrm{L}$. & romã & Sh & food; med (fr) & $\mathrm{E} / \mathrm{B}$ \\
\hline \multicolumn{5}{|l|}{ Malpighiaceae } \\
\hline Bunchosia armeniaca DC. & cereja & Sh & food (fr) & $\mathrm{E} / \mathrm{B}$ \\
\hline Malipighia emarginata Sessé \& Moc. Ex DC. & acerola & $\mathrm{Sh}$ & food (fr) & $\mathrm{E} / \mathrm{B}$ \\
\hline \multicolumn{5}{|l|}{ Malvaceae } \\
\hline Bombacopsis glabra (Pasq.) A. Rob. & castanha & $\mathrm{Ar}$ & cons (st) & $\mathrm{N} / \mathrm{B}$ \\
\hline Eriotheca sp. & paineira & $\mathrm{Ar}$ & cons (st); oth (fr) & $\mathrm{N} / \mathrm{B}$ \\
\hline Gossypium herbaceum L. & algodão & $\mathrm{Ar}$ & med (l) & $\mathrm{E} / \mathrm{F}$ \\
\hline Hibiscus rosa-sinensis $\mathrm{L}$. & mimo & Sh & orn (ap) & $\mathrm{E} / \mathrm{B}$ \\
\hline Malvaviscus arboreus Cav. & mimo & Sh & orn (ap) & $\mathrm{E} / \mathrm{B}$ \\
\hline Pachira aquatica Aubl. & castanha & $\mathrm{Ar}$ & cons (st) & $\mathrm{E} / \mathrm{B}$ \\
\hline Pseudobombax grandiflorum (Cav.) A Rob. & castanha & $\mathrm{Ar}$ & cons $(\mathrm{st})$ & $\mathrm{N} / \mathrm{B}$ \\
\hline Sida planicaulis Cav. & vassoura & $\mathrm{Hb}$ & med (l); tec (ap) & $\mathrm{N} / \mathrm{B}$ \\
\hline \multicolumn{5}{|l|}{ Marantaceae } \\
\hline Maranta divaricata Roscoe & araruta & $\mathrm{Hb}$ & food $(r)$ & $\mathrm{N} / \mathrm{B}$ \\
\hline
\end{tabular}


Table 2. Continuation.

\begin{tabular}{|c|c|c|c|c|}
\hline Family/Scientific name & Local name & Habit & Usage categories / Part used & $\mathrm{O} / \mathrm{Loc}$ \\
\hline \multicolumn{5}{|l|}{ Melastoforestceae } \\
\hline Leandra melastomoides Raddi & pixirica & $\mathrm{Ar}$ & fue (st); oth (fr) & $\mathrm{N} / \mathrm{F}$ \\
\hline Miconia cinnamomifolia Triana & jacatirão & $\mathrm{Ar}$ & cons $(\mathrm{st})$ & $\mathrm{N} / \mathrm{F}$ \\
\hline Miconia dodecandra (Desr.) Cogn. & pixirica & $\mathrm{Ar}$ & fue (st); oth (fr) & $\mathrm{N} / \mathrm{F}$ \\
\hline Miconia pusilliflora (DC.) Naudin & fruto-de-saíra & $\mathrm{Ar}$ & fue; cons (st) & $\mathrm{N} / \mathrm{F}$ \\
\hline Tibouchina gaudichaudianum Cogn. & chorão & $\mathrm{Ar}$ & fue (st); orn (wp) & $\mathrm{N} / \mathrm{F}$ \\
\hline Tibouchina sp. & quaresma & $\mathrm{Ar}$ & fue; cons; tec (st) & $\mathrm{N} / \mathrm{F}$ \\
\hline \multicolumn{5}{|l|}{ Meliaceae } \\
\hline Cedrela sp. & cedro & $\mathrm{Ar}$ & cons; tec (st) & $\mathrm{N} / \mathrm{F}$ \\
\hline Guarea macrophylla var. tuberculata Vahl. & cabacero & $\mathrm{Ar}$ & fue; cons; tec (st) & $\mathrm{N} / \mathrm{F}$ \\
\hline Trichilia trigolia var. ptilaefolia (A. Juss.) Penn. & guariçica-da-folha-miúda & $\mathrm{Ar}$ & cons $(\mathrm{st})$ & $\mathrm{N} / \mathrm{F}$ \\
\hline \multicolumn{5}{|l|}{ Moraceae } \\
\hline Artocarpus heterophyllus Lam. & jaca & $\mathrm{Ar}$ & food (fr); med (l) & $\mathrm{E} / \mathrm{B}$ \\
\hline Ficus glabra Vell. & figueira-branca; figueira & $\mathrm{Ar}$ & tec (r); oth (c) & $\mathrm{E} / \mathrm{B}$ \\
\hline Morus nigra $\mathrm{L}$. & amora & $\mathrm{Ar}$ & food (fr); med (l) & $\mathrm{E} / \mathrm{B}$ \\
\hline Sorocea guilleminiana Gaudich & espinheira-santa & Sh & med (l) & $\mathrm{N} / \mathrm{F}$ \\
\hline \multicolumn{5}{|l|}{ Musaceae } \\
\hline Musa X paradisiaca L. & banana & $\mathrm{Sh}$ & food (fr); med (l) & $\mathrm{E} / \mathrm{B}$ \\
\hline \multicolumn{5}{|l|}{ Myrtaceae } \\
\hline Calyptranthes strigipes O. Berg. & guamirim & $\mathrm{Ar}$ & fue; cons (st) & $\mathrm{N} / \mathrm{F}$ \\
\hline Eucalyptus sp. & eucalipto & $\mathrm{Ar}$ & med (l) & $\mathrm{E} / \mathrm{B}$ \\
\hline Eugenia tinguyensis Cambess. & araçarana-do-mato & $\operatorname{Ar}$ & fue; cons (st) & $\mathrm{N} / \mathrm{F}$ \\
\hline Eugenia uniflora $\mathrm{L}$. & pitanga & $\mathrm{Ar}$ & food (fr); med; vet (l); tec (st) & $\mathrm{N} / \mathrm{B}$ \\
\hline Gomidesia spectabilis (DC.) O. Berg & guamirim-vermelho & $\mathrm{Ar}$ & fue; cons (st) & $\mathrm{N} / \mathrm{F}$ \\
\hline Myrcia splendens (Sw.) DC. & cambucá-da-mata-virgem & $\mathrm{Ar}$ & cons $(s t)$ & $\mathrm{N} / \mathrm{F}$ \\
\hline Plinia edulis (O. Berg) Nied & cambucá & $\mathrm{Ar}$ & food (fr); fue (st); med (l) & $\mathrm{N} / \mathrm{B}$ \\
\hline Plinia trunciflora (O. Berg) Kausel & jabuticaba & $\mathrm{Ar}$ & food $(\mathrm{fr})$ & $\mathrm{N} / \mathrm{B}$ \\
\hline Psidium cattleianum Sabine & araçá & $\mathrm{Ar}$ & food (fr) & $\mathrm{N} / \mathrm{B}$ \\
\hline Psidium guajava $\mathrm{L}$. & goiaba & $\mathrm{Ar}$ & food (fr); med (l) & $\mathrm{E} / \mathrm{B}$ \\
\hline Syzygium jambos (L.) Alston & jambo & $\mathrm{Ar}$ & food (fr) & $\mathrm{E} / \mathrm{B}$ \\
\hline \multicolumn{5}{|l|}{ Myrsinaceae } \\
\hline Rapanea ferruginea (Ruiz \& Pav.) Mez & capororoca-da-folha-pequena & $\mathrm{Ar}$ & fue; cons (st) & $\mathrm{N} / \mathrm{F}$ \\
\hline \multicolumn{5}{|l|}{ Nyctaginaceae } \\
\hline Bougainvillea spectabilis Willd. & carramanchão & $\mathrm{Vi}$ & orn (ap) & $\mathrm{N} / \mathrm{B}$ \\
\hline \multicolumn{5}{|l|}{ Passifloraceae } \\
\hline Passiflora alata Curtis & maracujá & $\mathrm{Vi}$ & food (fr); med (l) & $\mathrm{N} / \mathrm{B}$ \\
\hline Passiflora edulis Sims & maracujá & $\mathrm{Vi}$ & food (fr); med (l) & $\mathrm{N} / \mathrm{B}$ \\
\hline \multicolumn{5}{|l|}{ Phyllanthaceae } \\
\hline Phyllanthus tenellus Roxb. & quebra-pedra & $\mathrm{Hb}$ & med (wp) & $\mathrm{N} / \mathrm{B}$ \\
\hline \multicolumn{5}{|l|}{ Piperaceae } \\
\hline Piper mollicomum Kunth & joão-borandí & $\mathrm{Vi}$ & med (l) & $\mathrm{N} / \mathrm{B}$ \\
\hline Piper nigrum $\mathrm{L}$. & pimenta-do-reino & $\mathrm{Vi}$ & food (fr) & $\mathrm{E} / \mathrm{B}$ \\
\hline Pothomorphe umbellata $\mathrm{L}$. & pariparoba & $\mathrm{Vi}$ & med (r) & $\mathrm{N} / \mathrm{F}$ \\
\hline \multicolumn{5}{|l|}{ Poaceae } \\
\hline Bambusa trinii Nees & taquara & $\mathrm{Ar}$ & tec $(\mathrm{st})$ & $\mathrm{N} / \mathrm{F}$ \\
\hline Bambusa tuldoides Munro & bambu-de-gaiola & $\mathrm{Ar}$ & fue; cons; tec (st) & $\mathrm{E} / \mathrm{B}$ \\
\hline Bambusa vulgaris Schrad. ex J.C. Wendl. & bambú-amarelo & $\mathrm{Ar}$ & fue; cons; orn; tec (st) & $\mathrm{E} / \mathrm{B}$ \\
\hline Cymbopogum citratus (DC.) Stapf. & capim-cidreira; capim-cidão & $\mathrm{Hb}$ & med (l) & $\mathrm{E} / \mathrm{B}$ \\
\hline Cymbopogum nardus (L.) Rendle & citronela & $\mathrm{Hb}$ & oth (l) & $\mathrm{E} / \mathrm{B}$ \\
\hline $\begin{array}{l}\text { Dendrocalamus asper (Schult. \& Schult. f.) } \\
\text { Backer ex K. Heyne }\end{array}$ & bambú-gigante & $\mathrm{Ar}$ & fue; cons; tec (st) & $\mathrm{E} / \mathrm{B}$ \\
\hline Eleusine indica (L.) Gaertn. & pé-de-galinha & $\mathrm{Hb}$ & med (wp) & $\mathrm{E} / \mathrm{B}$ \\
\hline Gynerium sagittatum (Aubl.) P. Beauv. & ubá & $\mathrm{Hb}$ & tec (st) & $\mathrm{N} / \mathrm{F} ; \mathrm{B}$ \\
\hline Imperata brasiliensis Trin. & sapê & $\mathrm{Hb}$ & cons (l) & $\mathrm{N} / \mathrm{F}$ \\
\hline Merostachys ternata Nees & taquara-de-lixa & Sh & tec $(\mathrm{st})$ & $\mathrm{N} / \mathrm{F}$ \\
\hline Phyllostachys pubescens Mazel ex J. Houz. & bambú-japonês & $\mathrm{Ar}$ & fue; cons; tec (st) & $\mathrm{E} / \mathrm{B}$ \\
\hline Saccharum officinarum $\mathrm{L}$. & cana & Sh & food $(s t)$ & $\mathrm{E} / \mathrm{B}$ \\
\hline Zea mays $\mathrm{L}$. & milho & $\mathrm{Hb}$ & food (i) & $\mathrm{E} / \mathrm{B}$ \\
\hline \multicolumn{5}{|l|}{ Polygalaceae } \\
\hline Polygala paniculata L. & arnica; gelol & $\mathrm{Hb}$ & med $(r)$ & $\mathrm{N} / \mathrm{B}$ \\
\hline
\end{tabular}


Table 2. Continuation

\begin{tabular}{|c|c|c|c|c|}
\hline Family/Scientific name & Local name & Habit & Usage categories / Part used & $\mathrm{O} / \mathrm{Loc}$ \\
\hline \multicolumn{5}{|l|}{ Rosaceae } \\
\hline Rosa canina $\mathrm{L}$. & rosa-branca & Sh & med; orn (fl) & $\mathrm{E} / \mathrm{B}$ \\
\hline Rubus rosifolius $\mathrm{Sm}$. & amora-de-espinho & $\mathrm{Hb}$ & med (wp) & $\mathrm{N} / \mathrm{F}$ \\
\hline \multicolumn{5}{|l|}{ Rubiaceae } \\
\hline Coffea arabica $\mathrm{L}$. & café & Sh & food; med (s) & $\mathrm{E} / \mathrm{B}$ \\
\hline \multicolumn{5}{|l|}{ Rutaceae } \\
\hline Citrus aurantium L. & laranja-china; laranja-lima & $\mathrm{Ar}$ & food (fr); med; vet (l) & $\mathrm{E} / \mathrm{B}$ \\
\hline Citrus deliciosa Ten. & laranja-crava; mixirica & $\mathrm{Ar}$ & food; oth (fr); med; vet (l) & $\mathrm{E} / \mathrm{B}$ \\
\hline Citrus limon (L.) Burm. f. & limão & $\mathrm{Ar}$ & food (fr); med; vet (l) & $\mathrm{E} / \mathrm{B}$ \\
\hline Citrus reticulata Blanco & pocã & $\mathrm{Ar}$ & food (fr); med (l) & $\mathrm{E} / \mathrm{B}$ \\
\hline Citrus sinensis (L.) Osbeck & laranja-baía; laranja & $\mathrm{Ar}$ & food (fr); med; vet (l) & $\mathrm{E} / \mathrm{B}$ \\
\hline Dictyoloma vandellianum Adr. Juss. & tingui & $\mathrm{Ar}$ & fue; tec (st) & $\mathrm{N} / \mathrm{F}$ \\
\hline Ruta graveolens $\mathrm{L}$. & arruda & $\mathrm{Hb}$ & med; rit; vet (l) & $\mathrm{E} / \mathrm{B}$ \\
\hline Zanthoxylum rhoifolium Lam. & peito-de-moça; espora-de-galo & $\mathrm{Ar}$ & tec $(\mathrm{st})$ & $\mathrm{N} / \mathrm{F}$ \\
\hline \multicolumn{5}{|l|}{ Sapindaceae } \\
\hline Cupania furfuraceae Radlk. & cubatã; cubatã-vermelho & $\mathrm{Ar}$ & fue; cons; tec (st) & $\mathrm{N} / \mathrm{F}$ \\
\hline Cupania oblongifolia Mart. & cubatã-branco & $\mathrm{Ar}$ & cons $(\mathrm{st})$ & $\mathrm{N} / \mathrm{F}$ \\
\hline \multicolumn{5}{|l|}{ Sapotaceae } \\
\hline Eclinusa ramiflora Mart. & guacá & $\mathrm{Ar}$ & tec (st) & $\mathrm{N} / \mathrm{F}$ \\
\hline Mimusops commersonii (G. Don) Engl. & abricó-da-praia & $\mathrm{Ar}$ & food (fr); oth (cr) & E/Bea \\
\hline \multicolumn{5}{|l|}{ Solanaceae } \\
\hline Capsicum frutescens $\mathrm{L}$. & pimenta; pimenta-malagueta & $\mathrm{Hb}$ & food (fr) & $\mathrm{E} / \mathrm{B}$ \\
\hline Lycopersicon esculentum Mill. & tomate & $\mathrm{Hb}$ & food (fr) & $\mathrm{E} / \mathrm{B}$ \\
\hline Solanum americanum Mill. & maria-preta & $\mathrm{Hb}$ & med (l) & $\mathrm{N} / \mathrm{B}$ \\
\hline Solanum sisymbrifolium Lam. & rebenta-cavalo & $\mathrm{Hb}$ & med (fr) & $\mathrm{N} / \mathrm{B}$ \\
\hline \multicolumn{5}{|l|}{ Trigoniaceae } \\
\hline Trigonia nivea var. nivea Camb. & cipó-de-paina & $\mathrm{Ar}$ & cons $(\mathrm{st}) ;$ tec $(\mathrm{s})$ & $\mathrm{N} / \mathrm{F}$ \\
\hline \multicolumn{5}{|l|}{ Ulmaceae } \\
\hline Trema micrantha (L.) Blume & candiúba & $\mathrm{Ar}$ & med (st) & $\mathrm{N} / \mathrm{F}$ \\
\hline \multicolumn{5}{|l|}{ Verbenaceae } \\
\hline Aegiphila sellowiana Cham. & capororoca-da-folha-grande & $\mathrm{Ar}$ & fue; cons (st) & $\mathrm{N} / \mathrm{F}$ \\
\hline Lippia alba (Mill.) N. E. Br. & erva-cidreira & $\mathrm{Hb}$ & med (l) & $\mathrm{N} / \mathrm{B}$ \\
\hline Stachytarpheta cayennensis (Rich.) Vahl. & gervão & $\mathrm{Hb}$ & med (wp) & $\mathrm{N} / \mathrm{B}$ \\
\hline Verbena tenera Spreng. & jardineira & $\mathrm{Hb}$ & orn (fl) & $\mathrm{N} / \mathrm{B}$ \\
\hline \multicolumn{5}{|l|}{ Vitaceae } \\
\hline Vitis labrusca L. & uva & $\mathrm{Vi}$ & food (fr) & $\mathrm{E} / \mathrm{B}$ \\
\hline \multicolumn{5}{|l|}{ Zingiberaceae } \\
\hline Hedychium coronarium J.Koenig & ciosa & $\mathrm{Hb}$ & med; orn (fl) & $\mathrm{E} / \mathrm{B}$ \\
\hline Zingiber officinale Roscoe & gengibre & $\mathrm{Hb}$ & med (st) & $\mathrm{E} / \mathrm{B}$ \\
\hline
\end{tabular}

island communities of Cardoso (SP) and Santa Catarina (SC), and recorded 111 native plants and 109 exotic ones. The authors inferred that the communities that mention a greater number of native species reflect an increased interaction with local vegetation, which can be explained by several complementary factors, such as the distance from larger urban centres, difficulty of access, dedication of the residents to tasks involving the environment, and the length of time residents had lived in the community.

The diagram of the sampling effort showed an ascending curve of significant relationships $\left(r^{2}=0.582 ; P<0.0001\right)$ between number of interviews and observed richness (Fig. 3 ), that is, an increase in sampling effort will probably result in an increase in richness. According to Amorozo (2002), knowledge of use of species is influenced by their availability, so in this way a region that has a very rich flora presents a greater number of species that may be used by the local population. The results we obtained corroborate this, which was observed by Amorozo (2002), since the Praia do Sono Caiçara community is situated in the Atlantic Forest area, an ecosystem recognized for its species-rich flora.

The prevalent habit among the useful plants was arboreal (95 spp.), 26\% of which belonged to the construction category, and $18 \%$ to the technological category. Other studies (Albuquerque et al. 2005; Cunha \& Albuquerque 2006; Alarcon \& Peixoto 2008) focussing on knowledge of forest tree species also showed that these uses are generally the most common. According to Christo et al. (2006), the high 
Table 3. Comparison of diversity indices of Shannon-Wiener $\left(H^{\prime}\right)$ in studies conducted in different coastal communities of the Brazilian coast. (NS: number of species; NI: number of informants, NC: number of use citations, $\mathrm{H}^{\prime}$ : Shannon-Wiener diversity index).

\begin{tabular}{|c|c|c|c|c|c|c|}
\hline \multirow{2}{*}{ Study site } & \multirow{2}{*}{ NS } & \multirow{2}{*}{ NI } & \multirow{2}{*}{ NC } & \multicolumn{2}{|c|}{$\mathrm{H}^{\prime}$} & \multirow{2}{*}{ References } \\
\hline & & & & base 10 & base $e$ & \\
\hline Gamboa, RJ & 90 & 58 & 558 & 1.65 & - & Figueiredo et al. 1993 \\
\hline Chalhaus, RJ & 75 & 42 & 482 & 1.53 & - & Figueiredo 1997 \\
\hline Arraial do Cabo, RJ & 68 & 15 & 444 & 1.78 & 4.10 & Fonseca-Kruel \& Peixoto 2004 \\
\hline Martim de Sá, RJ & 76 & 10 & 355 & 1.81 & - & Borges \& Peixoto 2009 \\
\hline Ilha de Búzios, SP & 128 & 56 & - & 1.57 & - & Begossi et al. 1993 \\
\hline Beach do Puruba, SP & 124 & 22 & 414 & 1.92 & - & Rossato et al. 1999 \\
\hline Sertão do Puruba, SP & 140 & 28 & 525 & 1.92 & - & Rossato et al. 1999 \\
\hline Picinguaba, SP & 216 & 83 & 1552 & 2.06 & - & Rossato et al. 1999 \\
\hline Casa de Farinha, SP & 108 & 18 & 393 & 1.85 & - & Rossato et al. 1999 \\
\hline Ilha de Vitória, SP & 57 & 11 & 195 & 1.61 & - & Rossato et al. 1999 \\
\hline Ponta do Almada, SP & 152 & 45 & 434 & 1.99 & 4.59 & Hanazaki et al. 2000 \\
\hline Beach de Camburí, SP & 162 & 57 & 541 & 1.98 & 4.57 & Hanazaki et al. 2000 \\
\hline Pereirinha e Itacuruçá, SP & 124 & 20 & 473 & 2.04 & - & Miranda \& Hanazaki 2008 \\
\hline Foles e Cambriú, SP & 86 & 31 & 340 & 1.83 & - & Miranda \& Hanazaki 2008 \\
\hline Naufragados, SC & 93 & 12 & 190 & 1.90 & - & Miranda \& Hanazaki 2008 \\
\hline Guaraqueçaba, PR & 445 & 90 & 3400 & 2.38 & 5.48 & Lima et al. 2000 \\
\hline Praia do Sono, RJ & 190 & 12 & 1341 & 2.18 & 5.03 & This study \\
\hline
\end{tabular}

percentage of tree species utilized, mainly in the construction and technological categories, reaffirms the importance of these types of uses of Atlantic Forest resources, while at the same time demands care, because the collection of these resources may cause damage to the conservation of species.

From the 190 species listed and collected, 61\% were sourced from the backyards of the interviewees, $37 \%$ from the forest, and $2 \%$ were found on the beach. The plants present in the backyards of the informants were used, as their main purpose, to promote health, nutrition and wellness, given most species from this environment were from the medicinal usage (76 spp.), nutritional (59 spp.) and ornamental (10 spp.) categories. Guarim Neto \& Carniello (2008) also found several plants in backyards with the potential to supply part of the basic needs of the family.

We found that backyards are usually tended by women and older family members, who interact more intensively with the children of the community. Thus, one can assume that the backyard is a potential space for the maintenance and transmission of traditional knowledge, given the opportunities for passing it to the next generation. Guarim Neto \& Carniello (2008) believe that, although having a reduced land area, the backyard brings together a vegetal grouping with intricate cultural manifestation, involving plant origin, handling and utilization.

Lima et al. (2000) report that high values of the ShannonWiener $\left(H^{\prime}\right)$ index, in general, relate to areas reasonably wellpreserved and associated with populations having significant ethnobotanical knowledge. The Shannon-Wiener $\left(H^{\prime}\right)$ index obtained in this study was 2.18 (base 10) and 5.03 (base e). When this index is compared with other communities studied, the Praia do Sono informants are the second high- est according to this parameter (Tab. 3), indicating that the specialists informants of Praia do Sono have great knowledge about the use of plant resources. However, it is important to note that other authors, with the exception of Fonseca-Kruel \& Peixoto (2004) and Borges \& Peixoto (2009), did not work with local specialists, but with a random sampling, superior numbers to the group we identified as the local specialists, as they attempted to cover the community as a whole.

Hanazaki et al. (2000), in analysing the rates of ShannonWiener $\left(H^{\prime}\right)$ index diversity in different Caiçara communities of the Atlantic Forest, were able to perceive some general patterns. The largest diversity was found in communities located on the continent, when compared to that found on the islands (Gamboa, Chalhaus, Búzios Island and Victoria Island). Rossato et al. (1999) discuss this tendency in the context of the theory of island biogeography (MacArthur \& Wilson 1967), whereby one expects to find a lower diversity of species on islands than in continental areas.

\section{Usage categories}

Grouping plants into nine categories according to their use revealed a high number of taxa indicated for medical purposes (89 spp.), followed by food use (67 spp.) and construction (48 spp.) (Tab. 2).

Of the 67 species listed for food uses, 48 were exotic and 19 native. Most of the plants (59 spp.) were present in the backyards of the informants, which demonstrated the large dependence of the residents of Praia do Sono on plant cultivation. The species known as ipi, or mandioca (Manihot esculenta), and the banana (Musa X paradisiaca), were the species cited by the most informants $(n=10)$. Several authors have found that Manihot esculenta is an important 

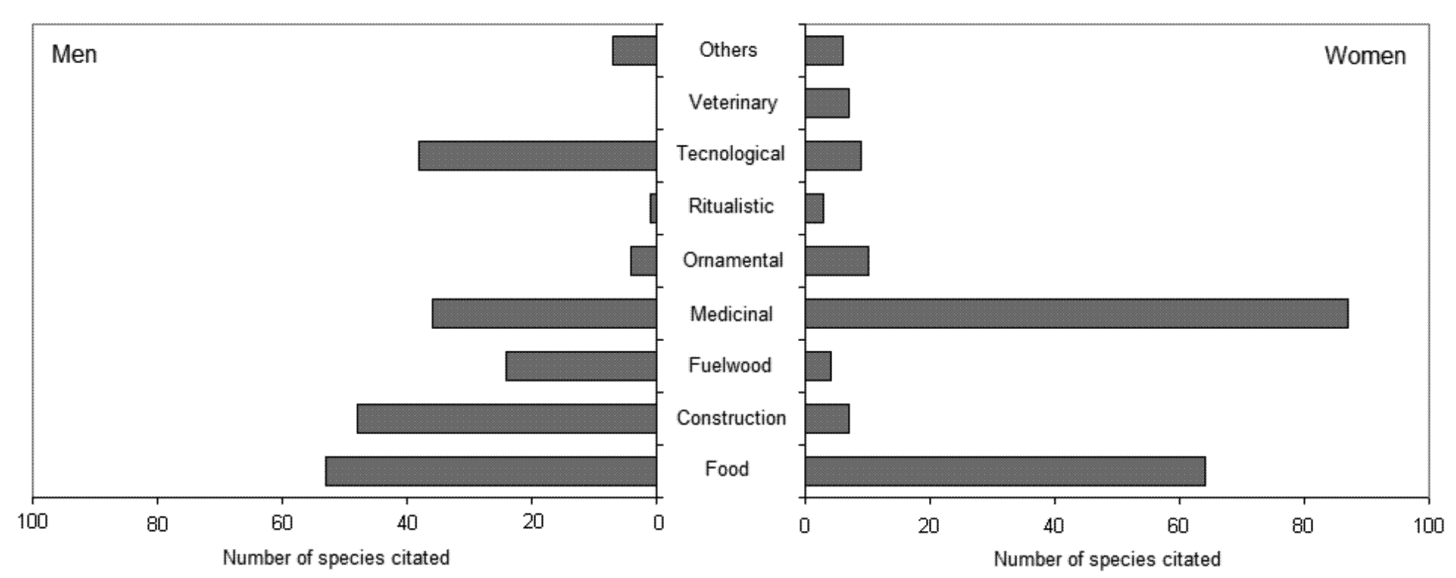

Figure 2. Distribution of the number of species indicated in each usage category by male and female informants of the Praia do Sono Caiçara community.

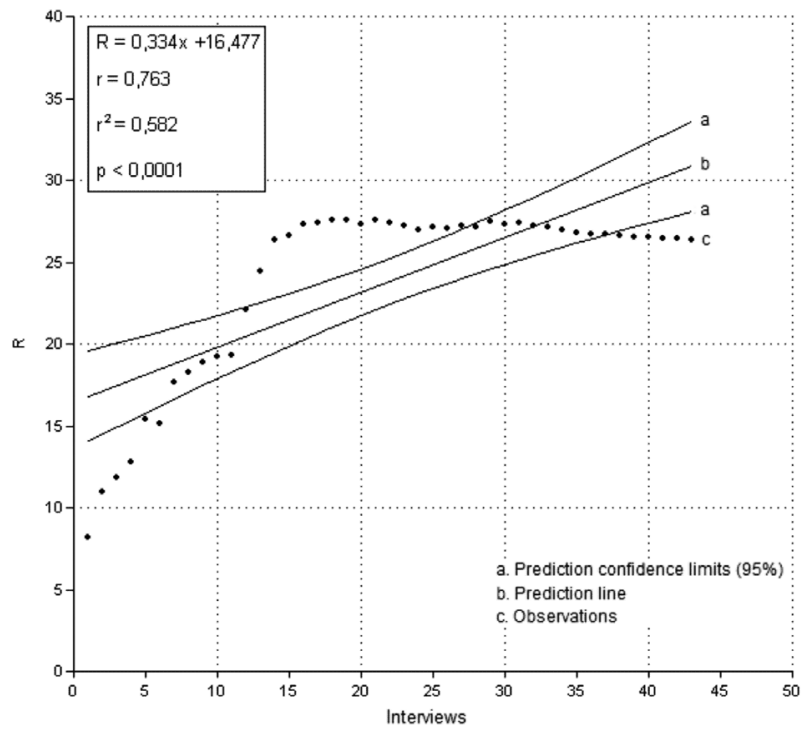

Figure 3. Graph of sampling effort related to the accumulation of species' richness according to the sequential insertion of interviews with informants from the Praia do Sono Caiçara community.

item in the Caiçara diet (Adams 2000; Hanazaki et al. 2000; Sanches 2004). Euterpe edulis is another food traditionally used by Caiçara communities (Sanches 2004). In this study, it was cited a significant number of times, as indicated by nine out of the 12 informants.

Of the 48 plants indicated for construction purposes, 42 were native and not cultivated by the local population. All six men interviewed, regardless of age, had a wide knowledge of the forest species used for construction, and this use is considered important in the culture and tradition of the Praia do Sono Caiçara community.

The traditional structure of the Caiçara house consists of walls of "pau-a-pique" or "estuque", sometimes whitewashed, thatched roofs and dirt floors. For the construction of walls,
Miconia cinnamomifolia and Pera glabrata were the best known species, because they were cited by all informants $(\mathrm{n}=12)$. Other native trees were indicated for this use, such as: Myrcia splendens, Mabea brasiliensis, Aegiphila sellowiana, Rapanea ferruginea, Sloanea guianensis, Trichilia trigolia var. ptilaefolia, Sparattosperma leucanthum, Hymenaea courbaril, Edulis edulis and Syagrus pseudococus. Species of bamboo were also mentioned in the context of the cross-patterned structures of the clay-covered intersecting walls; Bambusa tuldoides and Phyllostachys pubescens were the most recommended for this use. The species indicated for bracing were Davilla rugosa, Anthurium sp. and Heteropsis salicifolia, and Imperata brasiliensis as the roof coating.

The removal of wood from the forest follows certain criteria. Informants cited the optimum time as being during the waning moon, which, according to the informants, prevents the entry of insects and therefore increases its durability. It was also advised that one should not cut down the wood at the time of regrowth, because it is considered watery. This was also described by Adams (2000) in their studies of the Caiçaras population of the Atlantic Forest.

Often, wood extraction is in conflict with laws governing some Environmental Preservation Areas. However, it was observed that participants feel strongly, as expressed through their speech, about the importance of environmental conservation where they live: "... if the wood that is fallen in the forest hasn't rotted, I catch it, it is better than tearing and throwing"; "We know that if we don't take care of our own, it may end"; "When we need to get wood we go into the forest, but we take off just a little, for what we need in the moment. It's not like the industries that take off all and then the forest ends".

Twenty-four species are used as firewood for stoves, of which 19 are native and only five exotic. Pera glabrata was mentioned for this purpose by a larger number of respondents $(n=6)$.

The medicinal category contained the largest number of taxa, with a total of 89 species. This indicates the great 
importance that medicinal plants play in the daily life of local specialists. Based on a review of other ethnobotanical studies undertaken in Brazil, it is possible to perceive that the medicinal category is among the most frequently mentioned (Hanazaki \& Begossi 2006; Boscolo \& Senna-Valle 2008). This category was analysed in detail, due to the large number of plants mentioned in this group and the wealth of cultural and botanical data obtained in the field, as can be seen in Brito \& Senna-Valle (2011).

The ornamentation of houses and gardens in Praia do Sono is clearly an occupation undertaken by women. Thirteen plants were mentioned for ornamental use, of which 10 were collected in the backyards of the informants, demonstrating that the cultivation of ornamental plants in backyards is common. Eight species listed in this category were exotic, and five species from the local flora were used to decorate gardens and homes.

Only one woman informant commented about herbs used for ritualistic purposes; however, she only mentioned three species in this category: Mimosa pudica, Ruta graveolens and Leonotis nepetaefolia. In fact, this was not a subject openly discussed by the informants, perhaps due to prejudice or fear of discrimination. However, one informant was observed sweeping her house with a broom made from a herb known as "broom" (Sida planicaulis), where, while sweeping, she was softly singing a type of prayer. According to informants, they have used "since the old days of their elders" branches of Sida planicaulis and "rosemary broom" (Baccharis dracunculifolia) to clear the ground before building or planting. Although, they do not associate any ritualistic connotation with this practice.

The residents of Praia do Sono, especially women, devote part of their time to raising small animals, mainly chickens and ducks for eggs and meat production. It is very common for the juvenile chickens and ducks to be attacked by the lice that plague their nests and, to fight this disease, informants usually resorted to the use of plants. Seven vegetable species were indicated as suitable for pest control to diminish the parasites that attack chickens and ducks.

Forty species were cited in the technological category, comprising 32 native and eight exotic species. Dendrocalamus asper was cited by more respondents $(\mathrm{n}=9)$. This species is widely utilized in the structure of the poles on which fish and clothing are dried. Moreover, they are used to make different types of crafts, as well as barrage walls to prevent sand spillage.

Other plants from the forest mentioned in this category were: Zanthoxylum rhoifolium, used to make wooden spoon and socket; Bambusa trinii, whose fibres are used to manufacture guitar strings; the wood of Tabebuia cassinoides and Cecropia pachystachya, which is employed in the manufacture of tambourines; Sapium sp., known as tarumã, used to craft boats; Guarea macrophylla var. tuberculata, which children use to make their slingshots to play; and Tabebuia heptaphylla, which, due to its strong timber, was used for the pestle for pounding corn and coffee.

Species in the technological category are also found in the backyards of the informants, such as Ficus glabra, the root of which makes a good press for making mandioca flour, and Eugenia uniflora, the stem of which is used to make needles for weaving fishing nets.

The titipi, a flexible basket made of Heteropsis salicifolia and used to squeeze the grated mandioca, is one of the artifacts of indigenous culture incorporated into Caiçara daily life. Other plants were cited as being widely utilized to manufacture baskets. The balaios (also baskets), which serve to carry the mandioca and other products, are produced using Davilla rugosa, Anthurium sp., Bambusa trinii and Heteropsis salicifolia. The balainho (a small basket), made with Merostachys ternate, is used as a sewing box to store utensils for stitching.

Nowadays, houses, beach huts and the church are illuminated using candles, gas lamps and generators. However, older informants mentioned the use of a fifó, a torch made of bamboo (Dendrocalamus asper) that is soaked in the oil of Aleurites moluccana or Ricinus communis.

The fishermen use ink obtained from the bark of Schinus terebinthifolius and Tibouchina sp. to make fishing nets more resistant. The bark of the both species is boiled and the liquid is poured over the net which is nested within a canoe where it remains soaking for a day and is then dried on clothe lines in a horizontal position. This procedure is undertaken whenever a new net is to be used at sea, or when an older net begins to lose its color.

The main species indicated for manufacturing canoes, which are still produced by hand by by excavating tree trunks were: Ocotea sp., Cedrela sp., Piptadenia gonoacantha, Schizolobium parahyba, Cariniana estrellensis and Tachigali paratyensis. The island communities of Cardoso (SP) and Santa Catarina (SC), according to Miranda \& Hanazaki (2008), cited Schizolobium parahyba as the most important plant in the manufacture of canoes. Additionally, in relation to species used as tools for fishing, Anthurium sp., which are fairly resistant, serve as a cable to pull fishing nets, and Phyllostachys pubescens is used to make fishing rods. For oars, the preferred species are Eclinusa ramiflora and Tabebuia cassinoides. Crescentia cujete and Cedrela sp. are used to make bowls to draw water from canoes, and Cecropia pachystachya to serve as a bolster to roll the canoe in the sand. The stem of Gynerium sagittatum was cited as being excellent for baking fish.

The indications of "shade", "bird food" and "repellent" did not fit into any of the established categories of use. Therefore we decided to create a new category called "Others" to group plants that have a different utilization. Ten plants were placed in this category. Of these, Mimusops commersonii, Terminalia catappa and Ficus glabra were referred to as being useful for "shade"; Cymbopogum nardus and Citrus deliciosa as "repellent"; and Schinus terebinthifolius, Clusia lanceolata, Eriotheca sp. and the two species 
known as pixirica (Leandra melastomoides and Miconia dodecandra) as "bird food". Begossi et al. (1993) reported that the Caiçara community of Ilha de Búzios (SP) is known to place the fruits of Schinus terebinthifolius in traps to catch the Brazilian Tanager (Ramphocelus bresilius) and Rufousbellied Thrush (Turdus rufiventris).

From the results obtained in this study, it was concluded that the informants retain a broad knowledge of their surrounding plant resources, with which they maintain deep intimacy. The expansion of tourism and contact with residents from large urban centres are strong components in the degradation of this knowledge. Thus, its preservation, as a determinant factor in sustaining the cultural identity of this group, deserves attention and investment.

Considering that backyards play important ecological functions, while also contributing to the conservation of native species, growing plants in this space could be considered an important activity carried out by local populations in conserving the local environment.

In addition to the strong sentiment expressed by informants regarding the great value of maintaining the environment in which they live, local specialists are important players in the conservation of biological diversity, not only because of the knowledge they possess, but also because of the practices they perform. Therefore, the incorporation of their traditions regarding the uses of plant species is not only important, but is also fundamental in the in situ conservation strategies of the Conservation Unit.

\section{Acknowledgments}

We thank the Praia do Sono Caiçara community for sharing their knowledge; the Programa UFRJ/CENPES for financial support; the Programa de Pós-Graduação em Botânica do Museu Nacional for their support and attention; Maria Christina de Mello Amorozo, Fábio Pedro Souza de Ferreira Bandeira, Bárbara de Sá Haiad and Inês Machline Silva for their suggestions; Tânia Wendt for support, attention and revision of the text; Alexandre Christo for all of the quantitative help; and Maria Carmem Reis, Verônica Maioli and Gabriella Almeida-Azevedo for assistance in the field.

\section{References}

Adams, C. 2000. Caiçaras na Mata Atlântica: pesquisa científica versus planejamento e gestão ambiental. São Paulo, Annablume, FAPESP.

Alarcon, J.G.S.; Peixoto, A.L. 2008. Use of Terra Firme Forest by Caicubi caboclos, Middle Rio Negro, Amazonas, Brazil: a quantitative study. Economic Botany 62: 60-73.

Albuquerque, U.P.; Andrade, L.H.C. \& Silva, A.C.O. 2005. Use of plant resources in a seasonal dry forest (Northeastern Brazil). Acta Botanica Brasilica 19: 27-38.

Albuquerque, U.P.; Lucena, R.F.P. \& Cunha, L.V.F.C. (Orgs). 2008. Métodos e técnicas na pesquisa etnobotânica. Recife, NUPEEA.

Alexiades, M.N. \& Sheldon, J.W. (Eds). 1996. Selected guidelines for ethnobotanical research: a field manual. The New York Botanical Garden Press, New York Advances in Economic Botany 10: 1-306.
Amorozo, M.C.M. 2002. Uso e diversidade de plantas medicinais em Santo Antônio do Leverger, Mt, Brasil. Acta Botanica Brasilica 16: 189-203.

Begossi, A. 1996. Use of ecological methods in ethnobotany: diversity indices. Economic Botany 50: 280-289.

Begossi, A. 1998. Knowledge on the use of natural resources: contributions to localmanagement. Pp. 39-52. In: Hens, L.; Borden, R.; Suzuki, S. \& Caravello, G. (Eds.) Research in HumanEcology: an interdisciplinary overview. Bruxelas, The VUB Press.

Begossi, A.; Leitão-Filho, H.F. \& Richerson, P.J. 1993. Plant uses in a Brazilian coastal fishing community (Búzios island). Journal of Ethnobiology 13: 233-256.

Borges, R. \& Peixoto, A.L. 2009. Conhecimento e uso de plantas em uma comunidade caiçara do litoral sul do Estado do Rio de Janeiro, Brasil. Acta Botanica Brasilica 23: 769-779.

Boscolo, O.H. \& Senna-Valle, L. 2008. Plantas de uso medicinal em Quissamã, Rio De Janeiro, Brasil. Iheringia, Série Botânica 63: 263-277.

Brito, M.R. \& Senna-Valle, L. 2011. Plantas medicinais utilizadas na comunidade caiçara da Praia do Sono, Paraty, Rio de Janeiro, Brasil. Acta Botanica Brasilica 25: 363-372.

Christo, A.G.; Guedes-Bruni, R.R. \& Fonseca-Kruel, V.S. 2006. Uso de recursos vegetais em comunidades limítrofes à Reserva Biológica de Poço das Antas, Silva Jardim, Rj: estudo de caso na Gleba Aldeia Velha. Rodriguésia 57: 519-542.

Corrêa, M.P. (1926-1978). Dicionário das plantas úteis do Brasil e das exóticas cultivadas. Imprensa Nacional, Rio de Janeiro.

Cunha, L.V.F.C. \& Albuquerque, U.P. 2006. Quantitative ethnobotany in an Atlantic Forest fragment of Northeastern Brazil - implications to conservation. Environmental Monitoring and Assessment 114: 1-25.

Diegues, A.C. \& Arruda, R.S.V. 2001. Saberes tradicionais e biodiversidade no Brasil. Brasília, Ministério do Meio Ambiente.

Figueiredo, G.M. 1997. Ethnobotany of Atlantic Forest coastal communities: diversity of plant uses at Sepetiba Bay (SE Brazil). Human Ecology 25: 353-360.

Figueiredo, G.M.; Leitão-Filho, H.F. \& Begossi, A. 1993. Ethnobotany of Atlantic Forest coastal communities: diversity of plant uses in Gamboa (Itacuruçá Island, Brazil). Human Ecology 21: 419-430.

Fonseca-Kruel, V.S. \& Peixoto, A.L. 2004. Etnobotânica na Reserva Extrativista Marinha de Arraial do Cabo, RJ, Brasil. Acta Botanica Brasilica 18: 177-190.

Guarim Neto, G. \& Carniello, M.A. 2008. Quintais Mato-Grossenses: espaços de conservação e reprodução de saberes. Cáceres, Editora Unemat.

Hammer, O.; Harper, D.A.T. \& Ryan, P.D. 2001. Past: Paleontological statistics software package for education and data analysis. Paleontologia Electronica 4: 1-9.

Hanazaki, N. 2003. Comunidades, conservação e manejo: o papel do conhecimento ecológico local. Biotemas 16: 23-47.

Hanazaki, N.; Tamashiro, J.Y.; Leitão-Filho, H.F. \& Begossi, A. 2000. Diversity of plant uses in two caiçara communities from the Atlantic Forest coast, Brazil. Biodiversity and Conservation 9: 597-615.

Hanazaki, N. \& Begossi, A. 2006. Catfish and mullets: the food preferences and taboos of caiçaras (Southern Atlantic Forest coast, Brazil). Interciência 31: 123-129.

Laraia, R.B. 2002. Cultura: um conceito antropológico. $15^{\text {the }} \mathrm{ed}$. Rio de Janeiro, Jorge Zahar Editora.

Lima, R.X.; Silva, S.M.; Kuniyoshi, Y.S. \& Silva, L.B. 2000. Etnobiologia de comunidades continentais da Área de proteção ambiental de Guaraqueçaba, Paraná, Brasil. Etnoecológica 4: 33-55.

Loss, A.C.C. \& Silva, A.G. 2005. Comportamento de forrageio de aves nectarívoras de Santa Teresa, ES. Natureza on line 3: 48-52.

Macarthur, R.H. \& Wilson, E.O. 1967. The theory of island biogeography. Princeton, Princeton University Press.

Miranda, T.M. \& Hanazaki, N. 2008. Conhecimento e uso de recursos vegetais de restinga por comunidades das Ilhas do Cardoso (SP) e de Santa Catarina (SC), Brasil. Acta Botanica Brasilica 22: 203-215.

Phillips, O. \& Gentry, A.H. 1993. The useful plants of Tambopata, Peru: I. Statistical hypotheses tests with a new quantitative technique. Economic Botany 47: 15-32. 
Prance, G.T.; Baleé, W.; Boom, B.M. \& Carneiro, R.L. 1987. Quantitative ethnobotany and the case for conservation in Amazônia. Conservation Biology 1: 296-310.

Ribeiro, M.C.; Metzger, J.P.; Martensen, A.C.; Ponzoni, F.J. \& Hirota, M.M. 2009. The Brazilian Atlantic Forest: how much is left, and how is the remaining forest distributed? Implications for conservation. Biological Conservation 142: 1141-1153.

Rossato, S.C.; Leitão-Filho, H. \& Begossi, A. 1999. Ethnobotany of caiçaras of the Atlantic Forest coast (Brazil). Economic Botany 53: 387-395.

Sanches, R.A. 2004. Caiçaras e a Estação Ecológica de Juréia-Itatins: litoral sul de São Paulo. São Paulo, Annablume, FAPESP.
SEMADS-RJ (Secretaria de Estado de Meio Ambiente e Desenvolvimento Sustentável do Rio de Janeiro). 2001. Atlas das Unidades de Conservação da Natureza do Estado do Rio de Janeiro. Rio de Janeiro, Metalivros.

Stehmann, J.R.; Forzza, R.C.; Salino, A.; Sobral, M.; Da Costa, D.P. \& Kamino, L.H.Y. (Orgs). 2009. Plantas da Floresta Atlântica. Rio de Janeiro, Jardim Botânico do Rio de Janeiro.

Tabarelli, M.; Aguiar, A.V.; Ribeiro, M.C.; Metzger, J.P. \& Peres, C.A. 2010. Prospects for biodiversity conservation in the Atlantic Forest: lessons from aging human-modified landscapes. Biological Conservation 143: 2328-2340.

Whittaker, R.H. 1975. Communities and ecosystems. New York, Macmillan. 